\title{
Melatonin regulates the neurotransmitter secretion disorder induced by caffeine through the gut-brain axis in zebrafish (Danio rerio)
}

\author{
Qiannan Peng \\ Hainan University \\ Zeng Zhang \\ Hainan University \\ Dongxue Huo \\ Hainan University \\ Shuaiming Jiang \\ Hainan University \\ Chenchen Ma \\ Hainan University \\ Haibo Chang \\ Hainan University \\ Russel Reiter \\ University of Texas \\ Haitao Shi \\ Hainan University \\ Jiachao Zhang ( $\nabla$ zhjch321123@163.com ) \\ Hainan University https://orcid.org/0000-0001-8099-6749
}

\section{Research}

Keywords: Melatonin, Neurotransmitter, Intestinal Microbiota, Gut-Brain Axis, SCFAs, Probiotic

Posted Date: May 11th, 2020

DOI: https://doi.org/10.21203/rs.2.19122/v2

License: (c) (1) This work is licensed under a Creative Commons Attribution 4.0 International License.

Read Full License 


\section{Abstract}

Background: Melatonin has been widely used as a "probiotic agent" capable of producing strong neurotransmitter secretion regulatory effects. The probiotics related researches also provide the evidence of microbial interactions with the gut-brain axis for mental health. In the present study, a zebrafish neural hyperactivity model was established using caffeine induction, and the regulation and mechanism of melatonin and probiotic on zebrafish neurotransmitter secretion disorder were explored. To further address the challenge that if the gut microbes play an essential role in the regulation of neurotransmitter secretion disorder via a process that involves melatonin, the Germ-free (GF) zebrafish model was used to verify the hypothesis.

Results: Disorders of brain neurotransmitter secretion caused by caffeine, including that of dopamine (DA), $\mathrm{y}$-aminobutyric acid ( $\mathrm{\gamma}$-GABA), and 5-hydroxytryptamine (5-HT), were improved after interference treatment with melatonin or the probiotic. Metagenomic sequencing demonstrated that the melatonintreated zebrafish gradually restored their normal intestinal microbial structure, while probiotic supplementation may restructure a new microbiome. Additionally, supplementation with melatonin significantly regulated intestinal microbial functional features, which indicated the gut microbiota plays the key role in the function of melatonin. Based on this activity, a Germ-free zebrafish model was applied to verified our hypothesis in the following validation experiment. Validation experiment results revealed that the effect on the zebrafish in the GF group could not achieve that on the zebrafish in the melatonin group after adding the same dose of melatonin, and subsequent real-time PCR and metabolic pathway analysis confirmed the conclusion. Meanwhile, the content of acetic acid and propionic acid in the gut of not-germ-free zebrafish decreased after caffeine induction and increased significantly after melatonin treatment. However, no acetic or propionic acids were found, detected, changed as there are germ-free zebrafish.

Conclusions: In the present research, we identified the potential mechanism of melatonin regulation of neurotransmitter secretion disorder through the gut-brain axis, laying a foundation for exploring the prevention and treatment of some neuropsychiatric disorders by improving the intestinal microbiota.

\section{Background}

Good mental state is indispensable for optimal human health, and cerebral neurotransmitter dysfunction can often trigger a variety of diseases, including sleep disorders, endocrine dysfunction, neurasthenia, decreased immune function, etc., damaging health and even causing diseases ${ }^{[1,2]}$. Melatonin is an indolyl neuroendocrine molecule secreted by the pineal gland, which is called a physiological hypnagogue and plays a crucial role in maintaining mental health ${ }^{[3]}$. The intestine is a major site for melatonin production. Melatonin and its receptors are widely distributed in the gastrointestinal tract, with the amount of melatonin in the intestine being an estimate 400 times greater than that in the pineal gland and 10-100 times greater than that in the plasma ${ }^{[4,5]}$. As an endogenous synchronizing factor of the 
circadian clock, melatonin plays a role in regulating sleep-wake biorhythm, sedation, and hypnosis via interaction on specific receptors ${ }^{[6,7]}$.

The interaction between the intestinal microbiota, gastrointestinal tract, and central nervous system (CNS) at the level of specific nerves, hormones, and immunity is referred to as the gut-brain axis ${ }^{[8]}$. Clinical studies have indicated that there may be multiple means of communication between the brain and gut through the immune system, vagus nerves, or microbial regulation of neuroactive compounds ${ }^{[9]}$. Regarding the latter, intestinal bacteria produce short-chain fatty acids (SCFAs) and synthesize many hormones and brain neurotransmitters, thereby impacting brain function and host behavior ${ }^{[9]}$. Investigations using probiotics provide further evidence of microbial interactions with the gut-brain axis. Studies have shown that probiotics alter neurotransmitter secretion in the brain through the intestinal microbiota, thereby affecting the host mental state. For example, certain Lactobacillus species play an active role in modulating depression and stress-related behaviors, and research has indicated that probiotic Bifidobacterium may regulate the secretion of sex hormones in polycystic ovary syndrome patients through manipulating the intestinal microbiome ${ }^{[10,11]}$. The beneficial effects of probiotics in relieving neurological diseases also suggest a role for the microbiota in these diseases.

Zebrafish (Danio rerio) is a highly regarded vertebrate model system with multiple unique advantages. It has a conserved nervous system structure and the vibrant behavior pattern of vertebrates, which have been studied as a model for several neurological diseases, including Alzheimer's disease, Parkinson's disease, and other neurological diseases ${ }^{[12]}$. The gut tissue and function of zebrafish are also similar to those of mammals, and the microbiota structure of its gut is relatively simple, so it is easier than in mammals to clearly explain the relevant mechanism in the subsequent analysis of the gut-brain axis ${ }^{[13]}$.

Since melatonin mitigates the effects on multiple neurological diseases, gut-brain axis-related studies have also highlighted the role of the gut microbiota in neuromodulation. We hypothesized that the intestinal microbiota plays an important role in the improvement of neurotransmitter secretion disorders via melatonin. Additionally, we tested whether probiotics, which regulate the structure and metabolism of the intestinal microbiota, have the same effect as melatonin. To address these questions, we constructed a model of neural hyperactivity in zebrafish and modified this disorder by exogenous melatonin and probiotic administration. We utilized metagenomic approach to assess how exogenous melatonin and probiotics improve the neurotransmitter secretion disorder of zebrafish by adjusting the intestinal microbiota to better understand their roles and mechanisms in regulating neurotransmitter secretion disorders (Fig. 1A). Moreover, germ-free (GF) zebrafish were also used to verify the important role of the gut microbiota in the regulation of neurotransmitter secretion disorders by melatonin. In the present research, we identified the potential mechanism of melatonin regulation of neurotransmitter secretion disorder through the gut-brain axis, laying a foundation for exploring the prevention and treatment of some neuropsychiatric disorders by improving the intestinal microbiota.

\section{Results}




\section{Alteration of neurotransmitters in the zebrafish brain}

To investigate the effects of different treatments on neurotransmitter secretion in the zebrafish head, the levels of neural hyperactivity-related neurotransmitters in each group were determined (Fig. 1B). On day 1, the concentration of DA in the caffeine, melatonin and probiotic groups was significantly increased, while the concentrations of $\mathrm{y}$-GABA decreased, and the concentration of $5-\mathrm{HT}$ did not change significantly compared with those in the control group. The imbalance indicated that caffeine had successfully induced neurotransmitter secretion disorders in the zebrafish brain. After 14 days of equal treatment in different experimental groups, the level of the neurotransmitters in each group changed. In the caffeine group, the contents of DA, $\mathrm{Y}-\mathrm{GABA}$, and $5-\mathrm{HT}$ returned to almost normal levels. While the level of DA gradually returned to normal in the melatonin group. The $y$-GABA levels in the melatonin and probiotic groups were significantly higher than those in the control group $(p=0.045)$, and the concentration of $5-\mathrm{HT}$ in the melatonin group was markedly higher than that in the remaining three groups compared with the average level $(p=0.016)$.

\section{Effects of different treatments on altered zebrafish intestinal microbiota composition}

The distance based on weighted and unweighted UniFrac in Fig. 2A and Fig. S1 indicated that the distance between control and treatment groups narrowed gradually with the extension of the treatment time. The distance between the control and melatonin group on day 14 was the smallest, followed by the probiotic group, and then the caffeine group. PCA presented in Fig. S2 also demonstrated that the points representing caffeine, melatonin, and probiotic groups were highly disordered but different from the control group on day 1 , this indicated that the intestinal microbiota of zebrafish was disturbed by caffeine. After 14 days of corresponding treatment, the microbial community structure in each treatment group was similar to that in the control group after 14 days of treatment, the melatonin group had the highest similarity with the control group, followed by the probiotic and caffeine groups. Taken together, these data suggested that caffeine induction results in some destabilization in the zebrafish microbial community, while melatonin and probiotic supplementation regulated this effect.

\section{Comparison of the core microbiota and different microbes among the different treatment groups in each timepoint}

Based on the high-throughput 16S rRNA sequencing data, the effects of different treatments on the gut microbiota of zebrafish were evaluated, and the alpha and beta diversity of the zebrafish intestinal microbiota in different groups were compared on day 1and on day 14 (Table S1-S2). After 14 days of equal treatment, the Chao1, ACE, Shannon, and Simpson indices in the caffeine group were significantly higher than those in the control group. Moreover, supplementation with melatonin decreased intestinal microbial diversity compared with that in the caffeine group, while that in the probiotic group remained at a high level. Since different treatments had specific effects on the intestinal microbiota of zebrafish, we next selected the core genera accounting for more than $1 \%$ in each group after 14 days of treatment for comparative analysis (Fig. 2B), and further screened the differential genus at each time point to observe the variation of specific genus with time in each group (Fig. 3A and Fig. S3). Based on determining the 
differences in microbial composition, we further refined the differential genera among the groups on day 1 and day 14 (Table 1, Table S3 and Table S4). After two-day caffeine exposure, the intestinal microbial community structure of zebrafish was disturbed. Many of the differentially abundant bacterial genera showed a tendency to decrease compared with those in the control group $(p<0.05)$. After 14 days of different treatments, the intestinal microbiota composition of zebrafish in each experimental group changed in different ways (Table S5). There was no significant difference compared with those in the control group, indicating that melatonin supplementation gradually reversed caffeine-induced intestinal microbiota dysbiosis. For the probiotic group, we observed that probiotics introduced differentially abundant genera, and there were 6 differentially abundant genera compared with those in the caffeine group.

\section{The construction of the calm index of microbiota (CIM)}

Based on the above results, we found that both melatonin and probiotics regulate the intestinal microbiota. Then, we selected the differentially abundant genera to draw a ternary diagram (Fig. S4). As shown in Fig. 3B-D, most of the bacterial genera showed a downward trend compared with those in the control group at baseline after caffeine induction, while a few specific bacteria were enriched in the gut of the zebrafish induced by caffeine (Fig. $3 B$ ). On day 14 , the microbial community structure of each group showed some recovery, with the different bacteria decreasing (Fig. 3C).

To more directly observe the disturbance degree of caffeine interference, melatonin and probiotics supplement on intestinal microbiota, a "calm index of microbiota" was derived based on the distinguishing bacteria between control and treatment groups. Twenty-seven differentially abundant bacteria between the control and caffeine-induced zebrafish on day 1 were chosen as markers to construct the $\mathrm{CIM}_{\text {day } 1}$, and the CIM day 1 of each sample was calculated. The results indicated that a significant difference existed in the $\mathrm{CIM}_{\text {day } 1}$ between the control and the other three groups (Fig. 3D), and after recalculation on day 14, the $\mathrm{CIM}_{\text {day } 1}$ was improved (Fig. S5). Meanwhile, 11 differentially abundant genera among groups on day 14 were selected to build the CIM day 14 . Based on the CIM day 14, we observed that the gaps between the melatonin group, probiotic group, and control group were smaller than that in the caffeine group (Fig. 3D). This indicated that melatonin played a further role in promoting the recovery of the intestinal microbiome relative to that in the caffeine group. The formulas were concluded as follow:

\section{See formulas 1 and 2 in the supplementary files section.}

\section{Functional features of the intestinal microbiota in different treatments}

To investigate how caffeine interference and the supplementation of melatonin and probiotic affect metabolic pathways by regulating intestinal microbiota, we performed shotgun metagenomic sequencing for samples collected on day $14(n=20)$. We annotated the assembled genes and compared with KEGG database to further annotate the microbial metabolic pathways. The $Z$ score greater than 1.6 represent the significant differences. The results showed that different treatments significantly altered the 
metabolism of zebrafish intestinal microbiota (Fig. 4 and Fig. S6). Compared with normal zebrafish, arginine and proline metabolism (ko00330), glyoxylate and dicarboxylate metabolism (ko00630), propanoate metabolism (ko00640), butanoate metabolism (ko00650) and biotin metabolism (ko00780) were enriched in the caffeine group. Glycolysis / gluconeogenesis (ko00010), citrate cycle (ko00020), fructose and mannose metabolism (ko00051), lipopolysaccharide biosynthesis (ko00540), pyruvate metabolism (ko00620), thiamine metabolism (ko00730) and phosphotransferase system (ko02060) were enriched in the control group. However, the relative abundance of these microbial metabolic pathways in the zebrafish intestines of the melatonin and probiotic groups approached the normal zebrafish in the control group. Collectively, the caffeine induction and melatonin and probiotic supplementation markedly altered the metabolism of the zebrafish gut microbiota.

\section{Verification experiment through a GF zebrafish model}

To verify that the gut microbiota plays an essential role in the function of melatonin, a GF zebrafish model was used. Considering that the effects of neurotransmitter regulation were best in the melatonin group (Fig. S7B, up panel), we only retained four groups: the control, caffeine, GF, and melatonin groups. Compared with the results of the control group, the caffeine interference increased the hyperactivity and decreased the rest time of the zebrafish. Moreover, after adding the same dose of melatonin, the rest time which could reflect brain activity was significantly different between the GF and melatonin groups (Fig. S7B, bottom panel), a better effect was observed in the melatonin group. However, we did not observe any significant difference in the phenotype including body weight and body length among different groups

(Fig. S7A, C). Correspondingly, the neurotransmitter secretions Y-GABA, 5-HT, DA and their related synthetic genes were also found to be different (Fig. 5A-C). This confirmed the primary mediate role of the intestinal microbiota in the efficacy of melatonin. Notably, from both 2 experiments, we clearly observed the disorders of brain neurotransmitter secretion caused by caffeine, including that of dopamine (DA), $\mathrm{Y}^{-}$ aminobutyric acid ( $\mathrm{Y}-\mathrm{GABA}$ ), and 5-hydroxytryptamine (5-HT), were improved after interference treatment with melatonin. However, some discrepancies in the specific value of the same group were observed between the 2 experiments because of the zebrafish individual heterogeneity. Furthermore, six zebrafish genes related to the secretion of $\mathrm{Y}$-GABA, 5-HT and DA were selected for gene expression analysis, among which there were four groups of genes (PINK1, corresponding to DA; trh, corresponding to $\mathrm{Y}$-GABA; and trph2 and mao, corresponding 5-HT) showing high consistency with the results of zebrafish rest time, as shown in Fig. 5F-I.

To better explain the mechanism by which the intestine microbiota function in the process of melatonin regulation of neurotransmitter secretion disorders, we also determined the contents of metabolites in the gut of zebrafish. Since germ-free zebrafish had almost no intestinal microbiota, while the SCFAs were mainly produced by colonic anaerobic fermentation of undigested carbohydrates, SCFAs were not detected in the GF group here. Results showed that the contents of acetic acid and propionic acid in the intestine of zebrafish in the caffeine and melatonin groups decreased significantly after the treatment with caffeine, while the contents increased remarkably after melatonin treatment, resulting in levels that were obviously higher than those in the caffeine group (Fig. 5D, E). 


\section{Melatonin regulated neurotransmitter secretion disorders through the gut-brain axis}

Through the verification experiment, we highlighted the importance of intestinal microbiota in the melatonin-induced neurotransmitter regulation effects. Based on the Spearman's rank correlation coefficient, we constructed a network of the correlations among melatonin, genera, metabolic pathways, SCFAs, and neurotransmitters. As shown in Fig. 6A, supplementation with melatonin inhibited the growth of Shewanella, Deefgea and Enterobacteriaceae in the zebrafish gut, which were negatively correlated with propanoate metabolism, butanoate metabolism and phosphotransferase system, whereas a negative correlation was observed between Aeromonadaceae and Rhizobiales and melatonin. Additionally, it was found that melatonin supplementation elevated the levels of acetic acid and propionic acid, representing SCFAs, in the intestine, and the generated SCFAs further stimulated the secretion of neurotransmitters in the brain of zebrafish, accompanied by decreased levels of DA and elevated levels of $\mathrm{Y}$-GABA and $5-\mathrm{HT}$, which revealed the potential mechanism by which melatonin regulates neurotransmitter secretion disorders through the gut-brain axis (Fig. 6B).

\section{Discussion}

Melatonin, also known as the "darkness hormone", is essential for calming nerves and sleep regulation ${ }^{[14]}$. It has been reported that exogenous melatonin has a positive sleep-promoting effect on diurnal vertebrates, including humans ${ }^{[6]}$. Mounting evidence has also indicated that intestinal microorganisms affect brain function and psychological state of the host through the gut-brain axis. However, it is not clear whether and how melatonin regulates the neural hyperactivity state of the brain through the intestinal microbiota. Meanwhile, probiotics, as micro-ecological modulators of the intestine, can also have a major influence on the nervous system ${ }^{[15,16]}$, and whether they can regulate the neural excitability of the organism through the gut-brain axis is also worth further exploration. Herein, a zebrafish neural hyperactivity model was established to explore the regulatory effect and mechanism of melatonin and Lactobacillus plantarum HNU082 on neurotransmitter secretion disorder.

There is growing evidence suggesting that the gut microbiota produce and release many similar neurotransmitters to help regulate mood and promote sleep, while possibly affecting the CNS through the gut-brain axis ${ }^{[9,17]}$. In addition to the variation in the levels of neurotransmitters, the structure of the zebrafish intestinal microbiota is disordered after caffeine induction, potentially indicating that the imbalance of the intestinal microbiota may be related to the disorder of neurotransmitters. After 14 days treatment, the intestinal microbiota in the various groups gradually recovered, among which the similarity of the melatonin group and control group microbiota was the highest, while the caffeine group microbiota still had a large difference. Moreover, we found that Cetobacterium, the dominant genus in fish intestine ${ }^{[18]}$ which plays a crucial role in the synthesis of vitamin B12 and fermentation of carbohydrates and peptides ${ }^{[19,20]}$, was decreased significantly after caffeine induction and that self-healing and melatonin and probiotic supplementation failed to increase its content significantly. The bacterium Shewanella, which can restore only the insulin-secreting $\beta$ cells to the average level in sterile zebrafish ${ }^{[21]}$, 
also was significantly decreased after neural hyperactivity, while the content of Shewanella in the melatonin group increased compared with that in the self-healing caffeine group. Overall, melatonin supplementation may restore the original intestinal microbiota structure to a great extent, while the probiotic may tend to shape a new intestinal microbiota balance by introducing additional changes in the gut-specific genera. Previous studies have reported that melatonin supplementation can significantly affect the growth and metabolism of the intestinal microbiota, including the Enterobacter aerogenes which is sensitive to the neurohormone melatonin, as well as the amino acid metabolism and drug metabolism in the different hosts ${ }^{[22,23]}$. We also observed some genus, amino acid metabolism, carbohydrate metabolism, cofactor and vitamin metabolism, and membrane transport changed significantly in different groups. Current research has indicated that SCFAs are implicated in a range of neuropsychiatric disorders, with the ability to cross the blood-brain barrier, and are critical substances that connect the intestine to the central nervous system ${ }^{[24]}$. The metabolites of SCFAs, such as propanoate and butanoate, were found to be stimulated during self-healing to restore the neurotransmitter secretion levels in the caffeine group. Previous studies have shown that SCFAs can promote the production of colonic $5-\mathrm{HT}$ through their effects on enterochromaffin cells ${ }^{[25]}$, especially propanoate, which has been shown to alter the DA, 5-HT and GABA systems ${ }^{[26]}$. Furthermore, $5-\mathrm{HT}$ activates the vagus nerve. Additionally, $5-\mathrm{HT}$ is the precursor of melatonin, which may be a means to connect the brain to the intestinal microbes. Nevertheless, the SCFA metabolism in the melatonin and probiotic groups was significantly lower than that in the caffeine group. We hypothesized that supplementation might promote the production of SCFAs during neural hyperactivity regulation, which accelerates the recovery of neurotransmitter secretion in the brain and gradually regulates the hyperactive state. Therefore, the metabolism of SCFAs gradually tended towards normal in the melatonin and probiotic groups.

To verify whether gut microbes play a real role in the recovery of brain neurotransmitter secretion, germfree animals were used, this is a valid and frequently used in vivo experimental model for studies on the host gut microbiota in health and various diseases, such as cancer ${ }^{[27]}$, diabetes ${ }^{[28]}$, and cardiovascular ${ }^{[29]}$ and metabolic diseases ${ }^{[30]}$. Mice, zebrafish, dogs, chickens, pigs, and many types of other germ-free models have been appropriately used in various research studies to verify the link between the intestinal microbiota and some diseases ${ }^{[31-35]}$. As the importance of gut-brain axis in brain development has gradually been confirmed, germ-free animals have gradually become an accepted model for gut-brain axis studies, which has become an important research means and valuable experimental tool for the study of the intestinal microbiota in host behaviour and various brain diseases ${ }^{[36-39]}$. In our validation experiment, the recovery of neurotransmitters in the GF group did not reach the level as in the normal zebrafish after adding the same dose of melatonin, indicating the key role of the intestinal microbiota in the regulation process of neurotransmitter secretion by melatonin, which subsequent real-time PCR again confirmed. To better explain the mechanism by which the intestinal microbiota plays a role in the regulation of neurotransmitter secretion, the content of SCFAs in the gut of zebrafish was determined. The results showed that the contents of acetic acid and propionic acid decreased in the intestine of tested fish after caffeine induction, while the content increased after melatonin treatment and was higher than that in the fish school without melatonin treatment, corresponding to our previous speculation that 
melatonin may promote the secretion of neurotransmitters in the brain by affecting intestinal microbes and producing SCFAs.

In addition, we constructed the CIM for zebrafish based on a variety of specific genera in the zebrafish gut to quantify the degree of the effect of caffeine induction and subsequent melatonin and probiotic supplementation on the host gut microbiome which impacted the emotional state of zebrafish. Guo et al. ${ }^{[40]}$ have examined the gut microbiome between gout patients and healthy people and then built a microbial gout index based on specific bacteria for the diagnosis of gout. Microbial indicators are currently widely used in intestinal research. Herein, a significant gap was found in $\mathrm{CIM}_{\text {day } 1}$ between normal zebrafish and caffeine-treated zebrafish on day 1 , but the index showed a significant improvement at the end of the experiment. Moreover, it can also be seen from $\mathrm{CIM}_{\text {day }} 14$ that melatonin and the probiotic played a specific role in promoting the recovery of the intestinal microbiota of zebrafish relative to that of the caffeine group. The CIM established in this study may provide a new means for analysing neural hyperactivity disorder.

Collectively, melatonin supplementation gradually restored the normal homeostasis and metabolism of the intestine and promoted the production of SCFAs in the intestine by regulating the gut microbiota to accelerate the recovery of the neurotransmitter secretion level through the gut-brain axis. The present research revealed the potential mechanism by which melatonin regulates neurotransmitter secretion disorder through the gut-brain axis, supporting future development of psychotropic drugs targeting the intestinal microbiota.

\section{Materials And Methods}

\section{Main experimental design}

In experiment 1, fish were equally distributed into 24 glass tanks ( 15 fish/tank) after 14 days of acclimation, each containing $5 \mathrm{~L}$ of water. Then, tanks were randomly assigned to four groups to conduct the experiment (Fig. 1A): (1) control group: normal feed, regular filtered water; (2) caffeine group: $200 \mu \mathrm{M}$ caffeine was added to the water for two consecutive days to alter zebrafish activity. This concentration of caffeine was enough to cause neural hyperactivity disorders in zebrafish. Thereafter the zebrafish were maintained conventionally with standard feed and regular filter water; (3) melatonin group: $200 \mu \mathrm{M}$ caffeine was used for two consecutive days to change zebrafish activity, and then the fish were fed regularly, but the water was replaced with filtered water containing $1 \mu \mathrm{M}$ melatonin (melatonin powder was dissolved in anhydrous ethanol to $100 \mathrm{mM}$ and eventually diluted to a final concentration of $1 \mu \mathrm{M}$ ); and (4) probiotic group: zebrafish were treated with $200 \mu \mathrm{M}$ caffeine for two consecutive days to change the fish activity. Then they were maintained in regular filtered water, and fed with normal fish feed containing $10^{8} \mathrm{CFU}$ of Lactobacillus plantarum HNU082/g of feed every day. Unconsumed food, faeces, and dead fish were removed in a timely manner, and the corresponding fresh filtered water was replaced in each tank every day; the water for the melatonin group was replaced with filtered water containing 1 
$\mu \mathrm{M}$ melatonin to maintain the constant melatonin concentration, while the water for the other three groups was replaced with regular filtered water.

\section{Validation experimental design}

To verify the critical role of the intestinal microbiota in the secretion of related neurotransmitters, germfree (GF) zebrafish were also studied (Fig. 1A). The zebrafish were purchased from the China zebrafish resource centre, and the GF zebrafish model was established as previously reported ${ }^{[41]}$. Considering that the effects of neurotransmitter regulation were best in the melatonin treated fish, we used only 4 groups, the control, caffeine, GF and melatonin groups. The rearing and treatment conditions of the control, caffeine, and melatonin groups were the same as those in the experiment above. Two-day caffeine induction was conducted, and melatonin supplementation was performed for 14 days, as in the other treatment groups. Water and feed were sterilized by irradiation with ultraviolet radiation in advance. On day 1 and day 14 after two-day caffeine induction in experiment 2, the mean static time of the fish school in five minutes under night-time dark conditions in the control, caffeine, melatonin, and GF groups were observed and recorded the same as the first experiment.

\section{Zebrafish maintenance and feeding}

Adult zebrafish (Danio rerio) obtained from the China zebrafish resource centre were maintained in the aquatic experimental animal facility of the College of Food Science and Engineering, Hainan University. The experimental and animal care procedures were conducted following the recommendations of the Ethics Committee of Hainan University. All fish were housed for 14 days in $50 \mathrm{~L}$ tanks supplied with aerated and dechlorinated tap water to acclimate to the laboratory conditions before experiments. The acclimation period and experiments were performed at a temperature of $28 \pm 0.5^{\circ} \mathrm{C}$ and with a $14 \mathrm{~h}$ light/10 $\mathrm{h}$ dark cycle according to the standards of zebrafish care ${ }^{[42]}$. Commercial fodder was provided once daily at a fixed time, which was administered by $3 \%$ of the total weight of the fish in each tank. Dead zebrafish were removed promptly, and the cumulative mortality did not exceed $2 \%$ throughout the acclimation period. For the GF group, whole tanks were kept in the clean bench ventilated with filtered sterile air to maintain germ-free conditions until the end of the experiment. Sterile food was performed through ultraviolet sterilization. Water was treated by filter-sterilized system and irradiated overnight with ultraviolet in the clean bench to keep sterilize, and ensured that the temperature was maintained at room temperature during morning transfer (Pham et al., 2008).

\section{Sample collection for 16S rRNA and shotgun metagenomic sequencing and SCFAs determination}

Several zebrafish were randomly selected from each group on day $1,3,7$ and day 14 following the twoday caffeine induction. Fish were euthanized promptly by an ice water bath, and dissection was conducted vice the anus with an aseptic scalpel after the body was sterilized with $75 \%$ alcohol. The entire intestine from the esophagus to the anus was collected, and three fish were pooled as a sample, taking a 
total of 6 replicates in each group for DNA extraction and a total of 3 replicates in each group for shortchain fatty acid (SCFA) determination. After centrifugation at $12000 \mathrm{rpm}$ for $20 \mathrm{~min}$ at $4^{\circ} \mathrm{C}$, the head supernatants were subsequently collected and stored at $-80^{\circ} \mathrm{C}$ for ELISA. In the verification experiment, another 3 heads were collected as a sample, with a total of 3 replicates in each group, to conduct gene expression analysis by real-time PCR.

\section{Enzyme-linked immunosorbent assay (ELISA) and analysis of intestinal fatty acid metabolites by GC-MS}

After the samples were thawed, the supernatants of head tissue homogenate were assessed by an ELISA kit (Shanghai Xinyu Biotechnology, China) according to the manufacturer's instructions. The concentrations of dopamine (DA), $y$-aminobutyric acid ( $y$-GABA) and 5-hydroxytryptamine (5-HT) in head tissue were calculated based on the optical absorbance value at $450 \mathrm{~nm}$ (OD450) determined by a microplate reader. The fatty acid concentrations in zebrafish intestines were quantified by a gas chromatography-mass spectrometry (GC-MS) assay [43]. An Agilent 7890B gas chromatograph equipped with a mass spectrometer (Agilent 5977A, Agilent, Santa Clara, CA, USA) was used to conduct the analysis of the samples, and an HP-5 MS column $(30 \mathrm{~m} \times 0.25 \mathrm{~mm}, 0.25 \mu \mathrm{m}$, Agilent, Santa Clara, CA, USA) was employed to achieve separation. The oven program was as follows: $50^{\circ} \mathrm{C}$ for $1 \mathrm{~min}$, rise to $200^{\circ} \mathrm{C}$ at a rate of $10^{\circ} \mathrm{C} / \mathrm{min}$, held at $200^{\circ} \mathrm{C}$ for $5 \mathrm{~min}$, rise to $220^{\circ} \mathrm{C}$ at a rate of $5^{\circ} \mathrm{C} / \mathrm{min}$, held at $220^{\circ} \mathrm{C}$ for $10 \mathrm{~min}$, rise to $250^{\circ} \mathrm{C}$ at $15^{\circ} \mathrm{C} / \mathrm{min}$, and held at $250^{\circ} \mathrm{C}$ for $10 \mathrm{~min}$. The temperature of the inlet was held at $250^{\circ} \mathrm{C}$, and the mass range was scanned from $\mathrm{m} / \mathrm{z} 35-400$. The temperature of the ion source chamber and the transfer line was set at $230^{\circ} \mathrm{C}$ and $250^{\circ} \mathrm{C}$, respectively, with an electron energy of $70 \mathrm{eV}$.

\section{Real-time PCR for gene expression analysis}

The head of the fish was collected, and total RNA was isolated according to the manufacturer's instructions. A High Capacity cDNA Reverse Transcription Kit (Applied Biosystems, CA, USA) was used to conduct CDNA synthesis with the entire isolated RNA following the instructions. Real-time PCR was performed to determine the mRNA levels of the genes. The PCR mixtures $(20 \mu \mathrm{l})$ contained $10 \mu \mathrm{l}$ of SYBR green mix (TAKARA, Japan), $0.2 \mu \mathrm{M}$ each forward and reverse primer and $1 \mu \mathrm{l}$ of cDNA. The primers used were prepared with reference to a previous study ${ }^{[41,44-46]}$. A StepOne Plus System (Applied Biosystems) was used to conduct the real-time PCR under the condition of $10 \mathrm{~min}$ at $95^{\circ} \mathrm{C}$, followed by 40 cycles at $95^{\circ} \mathrm{C}$ for $15 \mathrm{~s}$ and $1 \mathrm{~min}$ at $60^{\circ} \mathrm{C}$, and ended by a dissociation step. StepOne Plus software was used to conduct the analysis of the results.

\section{DNA extraction from zebrafish intestine tissues and 16S ribosomal RNA gene sequencing}

Genomic DNA of zebrafish intestine was extracted using a QIAamp® DNA Stool Mini Kit (Qiagen, Hilden, Germany) following the manufacturer's instructions. The quality of all isolated DNA was assessed by A260/A280 using a NanoDrop instrument. Qualified DNA was preserved at $-20^{\circ} \mathrm{C}$ for subsequent analysis. The PCR amplification procedure was conducted as previously described ${ }^{[47]}$. A set of 6-nucleotide barcodes was added to the specific primers 338F and 806R to amplify the 16S rRNA V3-V4 region of the genomic DNA. An Agilent DNA 1000 kit combined with an Agilent 2100 Biologic Analyzer (Agilent 
Technologies, USA) was used to quantify the PCR products. Sequencing was performed using the Illumina MiSeq high-throughput sequencing platform by Shanghai Personal-Bio Corporation.

\section{Shotgun metagenomic sequencing and quality control}

The zebrafish samples on day 14 ( $n=20,5$ samples each group) were subjected to shotgun metagenomic sequencing by using an Illumina HiSeq 2500 instrument. Libraries were prepared with a fragment length of approximately $300 \mathrm{bp}$. Paired-end reads were generated using $100 \mathrm{bp}$ in the forward and reverse directions. The reads were trimmed using Sickle and were subsequently aligned to the human genome to remove the host DNA fragments.

\section{Functional annotation and metabolic pathway analysis}

The annotated amino acid sequences were aligned against the Kyoto Encyclopaedia of Genes and Genomes (KEGG) databases using BLASTp (e-value $\leq 1 \mathrm{e}-5$ with a bit-score higher than 60 ). The annotated sequences were assigned to the KEGG orthologue group (KO) according to the highest score. Reporter Zscores were calculated to reveal the differences in enriched metabolic pathways between the control and PCOS groups, as previously described. Accordingly, a reporter score of $>2.3$ ( $90 \%$ confidence according to the normal distribution) was used as a detection threshold to significantly differentiate between pathways ${ }^{[48]}$.

\section{Bioinformatic analyses and statistical analyses for figure construction}

A quality-control procedure was performed for the raw pair-end reads; bioinformatics analysis of highquality sequence data was conducted after removal of primer and barcode sequences by using the QIIME platform ${ }^{[49]}$. PyNAST ${ }^{[50]}$ was used to align the trimmed sequences, and those under $100 \%$ sequence identity were clustered using UCLUST ${ }^{[51]}$ to obtain a unique V3-V4 sequence set. On this basis, operational taxonomic units (OTUs) were clustered at a threshold level of $97 \%$ sequence identity, and the sequences with the highest frequency were selected as the representative sequences of OTUs. After ChimeraSlayer ${ }^{[52]}$ was employed to remove the potentially chimeric sequences in the representative set of OTUs, a representative sequence of each OTU was assigned to a taxonomic level by Ribosomal Database Project (RDP) ${ }^{[53]}$. A phylogenetic tree of OTUs was constructed by using Fast Tree ${ }^{[54]}$, and the alpha and beta diversity of bacterial communities were analysed on this basis. The sequence data reported in this paper have been deposited in the NCBI database (metagenomic data: PRJNA543612).

\section{Statistical analyses and figure construction}

Relatedness analysis was conducted with R Studio. Boxplot based on weighted and unweighted UniFrac distance were established using the ggplot2 package. Principal component analysis (PCA) was carried 
out by the ggord package, and the PHEATMAP package was used for cluster analysis and heatmap construction. Correlation analysis was calculated by using Spearman's rank correlation coefficient and visualized as a network by Cytoscape. Statistical significance was analysed with Mann-Whitney test or Ttest. Data are presented as the means \pm SEMs; a two-sided test was conducted, and $p<0.05$ was considered statistically significant. All statistical analyses were performed by GraphPad Prism, version 8 .

\section{Declarations}

\section{Acknowledgments}

We thank Xiaopeng Zhu and Hongyang Guo for their help in the experiment and data analysis.

\section{Funding}

This study was supported by the National Natural Science Foundation of China (No.31701577) and the Start-up Funding and the Scientific Research Foundation of Hainan University (No. KYQD1548).

\section{Authors' contributions}

HS and JZ conceived and designed the experiments. JZ, QP, ZZ, DH, CM, SJ and HC performed the experiments and analysed the data. QP, JZ, RR and HS wrote and revised the manuscript. All authors read and approved the final manuscript.

\section{Availability of data and materials}

The authors declare that the data supporting the findings of this study are available within the paper and its additional files. The sequence data reported in this manuscript have been deposited in the NCBI database (metagenomic data: PRJNA543612).

\section{Ethics approval and consent to participate}

The experimental and animal care procedures were conducted following the recommendations of the Ethics Committee of Hainan University.

\section{Competing interests}

The authors declare that they have no competing interests.

\section{Consent for publication}

Not applicable.

\section{Author details}


${ }^{1}$ College of Food Science and Technology, Hainan Key Laboratory for Sustainable Utilization of Tropical Bioresources, College of Tropical Crops, Hainan University, Haikou, Hainan province, 570228, China.

${ }^{2}$ Department of Cellular and Structural Biology, UT Health San Antonio, San Antonio, TX, USA

${ }^{3}$ Institute of Marine Science and Technology, Shandong University, Qingdao 266237, Shandong, P. R. China

\section{References}

1. Knutson KL, Spiegel K, Penev P, Van Cauter E: The metabolic consequences of sleep deprivation. Sleep Med Rev 2007, 11:163-178.

2. Spaeth AM, Dinges DF, Goel N: Effects of Experimental Sleep Restriction on Weight Gain, Caloric Intake, and Meal Timing in Healthy Adults. Sleep 2013, 36:981-990.

3. Claustrat B, Leston J: Melatonin: Physiological effects in humans. Neurochirurgie 2015, 61:77-84.

4. Bubenik GA: Localization, physiological significance and possible clinical implication of gastrointestinal melatonin. Biol Signals Recept 2001, 10:350-366.

5. Dubocovich ML, Markowska M: Functional MT1 and MT2 melatonin receptors in mammals. Endocrine 2005, 27:101-110.

6. Gandhi AV, Mosser EA, Oikonomou G, Prober DA: Melatonin is required for the circadian regulation of sleep. Neuron 2015, 85:1193-1199.

7. Keijzer H, Smits MG, Duffy JF, Curfs LM: Why the dim light melatonin onset (DLMO) should be measured before treatment of patients with circadian rhythm sleep disorders. Sleep Med Rev 2014, 18:333-339.

8. Cryan JF, O'Mahony SM: The microbiome-gut-brain axis: from bowel to behavior. Neurogastroenterol Motil 2011, 23:187-192.

9. Strandwitz P: Neurotransmitter modulation by the gut microbiota. Brain Res 2018, 1693:128-133.

10. Bravo JA, Forsythe P, Chew MV, Escaravage E, Savignac HM, Dinan TG, Bienenstock J, Cryan JF: Ingestion of Lactobacillus strain regulates emotional behavior and central GABA receptor expression in a mouse via the vagus nerve. Proc Natl Acad Sci U S A 2011, 108:16050-16055.

11. Zhang J, Sun Z, Jiang S, Bai X, Ma C, Peng Q, Chen K, Chang H, Fang T, Zhang H: Probiotic Bifidobacterium lactis V9 Regulates the Secretion of Sex Hormones in Polycystic Ovary Syndrome Patients through the Gut-Brain Axis. mSystems 2019, 4.

12. Kalueff AV, Stewart AM, Gerlai R: Zebrafish as an emerging model for studying complex brain disorders. Trends Pharmacol Sci 2014, 35:63-75.

13. de Abreu MS, Giacomini A, Sysoev M, Demin KA, Alekseeva PA, Spagnoli ST, Kalueff AV: Modeling gut-brain interactions in zebrafish. Brain Res Bull 2019, 148:55-62.

14. Dawson D, Encel N: Melatonin and sleep in humans. J Pineal Res 1993, 15:1-12. 
15. Mairesse J, Rodenas CLG, Silletti V, Cassano T, Bergonzelli GE, Maccari S: Lactobacillus Reuteri Dsm 17938 and Bifidobacterium Longum Atcc Baa-999 Normalize Sleep Patterns in Prenatal Stress Rats. Pediatric Research 2011, 70:797-797.

16. Takada M, Nishida K, Gondo Y, Kikuchi-Hayakawa H, Ishikawa H, Suda K, Kawai M, Hoshi R, Kuwano Y, Miyazaki K, Rokutan K: Beneficial effects of Lactobacillus casei strain Shirota on academic stressinduced sleep disturbance in healthy adults: a double-blind, randomised, placebo-controlled trial. Beneficial Microbes 2017, 8:153-162.

17. Gao T, Wang Z, Dong Y, Cao J, Lin R, Wang X, Yu Z, Chen Y: Role of melatonin in sleep deprivationinduced intestinal barrier dysfunction in mice. J Pineal Res 2019:e12574.

18. Larsen AM, Mohammed HH, Arias CR: Characterization of the gut microbiota of three commercially valuable warmwater fish species. $J$ App/ Microbio/ 2014, 116:1396-1404.

19. Finegold SM, Vaisanen ML, Molitoris DR, Tomzynski TJ, Song Y, Liu C, Collins MD, Lawson PA: Cetobacterium somerae sp. nov. from human feces and emended description of the genus Cetobacterium. Syst Appl Microbio/ 2003, 26:177-181.

20. Tsuchiya C, Sakata T, Sugita H: Novel ecological niche of Cetobacterium somerae, an anaerobic bacterium in the intestinal tracts of freshwater fish. Lett Appl Microbio/ 2008, 46:43-48.

21. Hill JH, Franzosa EA, Huttenhower C, Guillemin K: A conserved bacterial protein induces pancreatic beta cell expansion during zebrafish development. Elife 2016, 5.

22. Ren WK, Wang P, Yan JM, Liu G, Zeng BH, Hussain T, Peng C, Yin J, Li TJ, Wei H, et al: Melatonin alleviates weanling stress in mice: Involvement of intestinal microbiota. Journal of Pineal Research 2018, 64 .

23. Paulose JK, Wright JM, Patel AG, Cassone VM: Human Gut Bacteria Are Sensitive to Melatonin and Express Endogenous Circadian Rhythmicity. PLoS One 2016, 11:e0146643.

24. Dalile B, Van Oudenhove L, Vervliet B, Verbeke K: The role of short-chain fatty acids in microbiota-gutbrain communication. Nature Reviews Gastroenterology \& Hepatology 2019, 16:461-478.

25. Reigstad CS, Salmonson CE, Rainey JF, Szurszewski JH, Linden DR, Sonnenburg JL, Farrugia G, Kashyap PC: Gut microbes promote colonic serotonin production through an effect of short-chain fatty acids on enterochromaffin cells. Faseb Journal 2015, 29:1395-1403.

26. El-Ansary AK, Ben Bacha A, Kotb M: Etiology of autistic features: the persisting neurotoxic effects of propionic acid. J Neuroinflammation 2012, 9:74.

27. Pollard M, Sharon N: Chemotherapy of Neoplastic Diseases in Germfree Mice. Mount Sinai Journal of Medicine 1974, 41:306-310.

28. Alam C, Bittoun E, Bhagwat D, Valkonen S, Saari A, Jaakkola U, Eerola E, Huovinen P, Hanninen A: Effects of a germ-free environment on gut immune regulation and diabetes progression in non-obese diabetic (NOD) mice. Diabetologia 2011, 54:1398-1406.

29. Wang ZN, Klipfell E, Bennett BJ, Koeth R, Levison BS, Dugar B, Feldstein AE, Britt EB, Fu XM, Chung $\mathrm{YM}$, et al: Gut flora metabolism of phosphatidylcholine promotes cardiovascular disease. Nature 2011, 472:57-U82. 
30. Nicholson JK, Holmes E, Kinross J, Burcelin R, Gibson G, Jia W, Pettersson S: Host-gut microbiota metabolic interactions. Science 2012, 336:1262-1267.

31. Harding JC, Ellis JA, McIntosh KA, Krakowka S: Dual heterologous porcine circovirus genogroup 2a/2b infection induces severe disease in germ-free pigs. Vet Microbio/ 2010, 145:209-219.

32. Furuse $\mathrm{M}$, Okumura J: Nutritional and physiological characteristics in germ-free chickens. Comp Biochem Physiol A Physiol 1994, 109:547-556.

33. Nance FC, Cain JL: Studies of hemorrhagic pancreatitis in germ-free dogs. Gastroenterology 1968, 55:368-374.

34. Melancon E, Gomez De La Torre Canny S, Sichel S, Kelly M, Wiles TJ, Rawls JF, Eisen JS, Guillemin K: Best practices for germ-free derivation and gnotobiotic zebrafish husbandry. Methods Cell Bio/2017, 138:61-100.

35. Al-Asmakh M, Zadjali F: Use of Germ-Free Animal Models in Microbiota-Related Research. Journal of Microbiology and Biotechnology 2015, 25:1583-1588.

36. Zheng P, Zeng BH, Liu ML, Chen JJ, Pan JX, Han Y, Liu YY, Cheng K, Zhou CJ, Wang HY, et al: The gut microbiome from patients with schizophrenia modulates the glutamate-glutamine-GABA cycle and schizophrenia-relevant behaviors in mice. Science Advances 2019, 5.

37. Horne R, Foster JA: Metabolic and Microbiota Measures as Peripheral Biomarkers in Major Depressive Disorder. Frontiers in Psychiatry 2018, 9.

38. Borre YE, Moloney RD, Clarke G, Dinan TG, Cryan JF: The Impact of Microbiota on Brain and Behavior: Mechanisms \& Therapeutic Potential. Microbial Endocrinology: The Microbiota-Gut-Brain Axis in Health and Disease 2014, 817:373-403.

39. Neufeld KM, Kang N, Bienenstock J, Foster JA: Reduced anxiety-like behavior and central neurochemical change in germ-free mice. Neurogastroenterology and Motility 2011, 23.

40. Guo Z, Zhang J, Wang Z, Ang KY, Huang S, Hou Q, Su X, Qiao J, Zheng Y, Wang L, et al: Intestinal Microbiota Distinguish Gout Patients from Healthy Humans. Sci Rep 2016, 6:20602.

41. Ding QW, Zhang Z, Ran C, He SX, Yang YL, Du ZY, Zhang JX, Zhou ZG: The Hepatotoxicity of Palmitic Acid in Zebrafish Involves the Intestinal Microbiota. Journal of Nutrition 2018, 148:1217-1228.

42. Westerfield M: The zebrafish book : a guide for the laboratory use of zebrafish (Brachydanio rerio). Eugene, OR: M. Westerfield; 1993.

43. Wei X, Song M, Yin X, Schuschke DA, Koo I, McClain CJ, Zhang X: Effects of Dietary Different Doses of Copper and High Fructose Feeding on Rat Fecal Metabolome. J Proteome Res 2015, 14:40504058.

44. Borrelli L, Aceto S, Agnisola C, De Paolo S, Dipineto L, Stilling RM, Dinan TG, Cryan JF, Menna LF, Fioretti A: Probiotic modulation of the microbiota-gut-brain axis and behaviour in zebrafish. Sci Rep 2016, 6:30046.

45. Moriya S, Tan VP, Yee AK, Parhar IS: pink1, atp13a2 and uchl1 expressions are affected by inflammation in the brain. Neurosci Lett 2019, 708:134330. 
46. Teng M, Zhu W, Wang D, Yan J, Qi S, Song M, Wang C: Acute exposure of zebrafish embryo (Danio rerio) to flutolanil reveals its developmental mechanism of toxicity via disrupting the thyroid system and metabolism. Environ Pollut 2018, 242:1157-1165.

47. Dethlefsen L, Relman DA: Incomplete recovery and individualized responses of the human distal gut microbiota to repeated antibiotic perturbation. Proc Natl Acad Sci U S A 2011, 108 Suppl 1:45544561.

48. Qin N, Yang F, Li A, Prifti E, Chen Y, Shao L, Guo J, Le Chatelier E, Yao J, Wu L, et al: Alterations of the human gut microbiome in liver cirrhosis. Nature 2014, 513:59-64.

49. Caporaso JG, Kuczynski J, Stombaugh J, Bittinger K, Bushman FD, Costello EK, Fierer N, Pena AG, Goodrich JK, Gordon JI, et al: QIIME allows analysis of high-throughput community sequencing data. Nat Methods 2010, 7:335-336.

50. Caporaso JG, Bittinger K, Bushman FD, DeSantis TZ, Andersen GL, Knight R: PyNAST: a flexible tool for aligning sequences to a template alignment. Bioinformatics 2010, 26:266-267.

51. Edgar RC: Search and clustering orders of magnitude faster than BLAST. Bioinformatics 2010, 26:2460-2461.

52. Haas BJ, Gevers D, Earl AM, Feldgarden M, Ward DV, Giannoukos G, Ciulla D, Tabbaa D, Highlander SK, Sodergren E, et al: Chimeric 16S rRNA sequence formation and detection in Sanger and 454pyrosequenced PCR amplicons. Genome Res 2011, 21:494-504.

53. Cole JR, Chai B, Farris RJ, Wang Q, Kulam-Syed-Mohideen AS, McGarrell DM, Bandela AM, Cardenas E, Garrity GM, Tiedje JM: The ribosomal database project (RDP-II): introducing myRDP space and quality controlled public data. Nucleic Acids Res 2007, 35:D169-172.

54. Price MN, Dehal PS, Arkin AP: FastTree: computing large minimum evolution trees with profiles instead of a distance matrix. Mol Biol Evol 2009, 26:1641-1650.

\section{Table}

Table 1. Differentially abundant genera between the control and each treatment group, namely, the caffeine, melatonin and probiotic groups, on day 14. 


\begin{tabular}{llllll}
\hline Genus & \multicolumn{2}{l}{ Mean abundance (\%) } & Enriched & \multicolumn{2}{c}{$P$-value } \\
\hline Control vs Caffeine & Control & Caffeine & Enriched & T test & M-W test \\
Cetobacterium & 22.7045 & 0.0013 & Control & 0.0001 & 0.0033 \\
\hline Unclassified_Neisseriaceae & 3.5683 & 0.4364 & Control & 0 & 0.0039 \\
\hline Shewanella & 2.6087 & 0.5769 & Control & 0.0002 & 0.0039 \\
\hline Unclassified_Enterobacteriaceae & 44.6963 & 27.097 & Control & 0.0001 & 0.0039 \\
\hline Unclassified_CK.1C4.19 & 10.4126 & 0 & Control & 0.0007 & 0.0021 \\
\hline Agrobacterium & 0.0386 & 1.9815 & Caffeine & 0.0007 & 0.0039 \\
\hline Unclassified_Rhizobiales & 0.0597 & 4.0318 & Caffeine & 0.0006 & 0.0039 \\
\hline Rhodobacter & 0.5349 & 20.7419 & Caffeine & 0.0003 & 0.0039 \\
\hline Unclassified_Rhodobacteraceae & 0.0372 & 1.4068 & Caffeine & 0 & 0.0039 \\
\hline Unclassified_Aeromonadaceae & 7.1575 & 21.1868 & Caffeine & 0.0005 & 0.0039 \\
\hline Control vs Melatonin & Control & Melatonin & Enriched & T test & M-W test \\
\hline Cetobacterium & 22.7045 & 0.0013 & Control & 0.0001 & 0.0028 \\
\hline Unclassified_Neisseriaceae & 3.5683 & 1.3105 & Control & 0.0077 & 0.0374 \\
\hline Unclassified_CK.1C4.19 & 10.4126 & 0.7239 & Control & 0.0008 & 0.0039 \\
\hline Unclassified_Rhizobiales & 0.0597 & 1.9050 & Caffeine & 0.0002 & 0.0039 \\
\hline Rhodobacter & 0.5349 & 28.238 & Caffeine & 0.0013 & 0.0039 \\
\hline Unclassified_Rhodobacteraceae & 0.0372 & 1.3113 & Caffeine & 0.0004 & 0.0039 \\
\hline Control vs Probiotic & Control & Probiotic & Enriched & T test & M-W test \\
\hline Cetobacterium & 22.7045 & 0.0013 & Control & 0.0001 & 0.0033 \\
\hline Unclassified_Neisseriaceae & 3.5683 & 1.0876 & Control & 0 & 0.0039 \\
\hline Shewanella & 2.6087 & 1.2601 & Control & 0.0014 & 0.0039 \\
\hline Unclassified_Enterobacteriaceae & 44.6963 & 34.9469 & Control & 0.0011 & 0.0104 \\
\hline Unclassified_CK.1C4.19 & 10.4126 & 0.6230 & Control & 0.0008 & 0.0039 \\
\hline Agrobacterium & 0.0386 & 4.2222 & Probiotic & 0.0054 & 0.0039 \\
\hline Unclassified_Rhizobiales & 0.0597 & 2.5901 & Probiotic & 0.0009 & 0.0039 \\
\hline Rhodobacter & 0.5349 & 21.1115 & Probiotic & 0 & 0.0039 \\
\hline Unclassified_Rhodobacteraceae & 0.0372 & 2.7394 & Probiotic & 0.0003 & 0.0039 \\
\hline Deefgea & 0.4297 & 1.2822 & Probiotic & 0.0068 & 0.0039 \\
\hline
\end{tabular}

Note: M-W test represents the Mann-Whitney test.

\section{Additional Files}

Additional file 1: Figure S1. The distance based on the weighted UniFrac between the control and three different treatment groups display the difference of the intestinal microbiota among different groups on day 1 (A), day 3 (B), day 7 (C) and day 14(D), which include control vs caffeine, control vs melatonin, and control vs probiotic. Figure S2. PCA based on the OTU in the gut. Each dot represents the composition of the gut microbiota of a sample, and different color represent zebrafish gut samples at different time points and groups. Figure S3. The significantly difference genera of each group in every time point. Figure S4. Heatmap constructed using the significantly different microbes among different groups at the genus level. The depth degree of color represents the relative abundance of the specific genus (blue indicates a small number and red indicates a large number). Figure S5. Variation of $\mathrm{CIM}_{\text {day } 1}$ on the day 14 in each group. Each dot represents a sample, and different color represent different groups. Figure S6. Functional features of the intestinal microbiota in different treatments. Figure S7. The performance in phenotype including $(A, C)$ fish body weight, fish body length and $(B)$ the rest of time in the night per 5 minutes 
among the groups in the first (up panel) and the verification experiment (bottom panel) on day 14. *, $p<0.05 ; * \star \star, p<0.001$. Table S1. Alpha diversity of zebrafish intestinal microbial communities in different treatment groups on day 14 . Table S2. Alpha diversity of zebrafish intestinal microbial communities in different treatment groups on day 1. Table S3. Differential genera between the control and model groups on the day 1 , including the caffeine, melatonin and probiotic groups. Table S4. Differential genera between the caffeine and each treatment group on the day 14 , including the melatonin and probiotic groups. Table S5. UniFrac distance based on the intestinal microbial taxonomic profile between the samples in the treatment group and the control group.

\section{Figures}

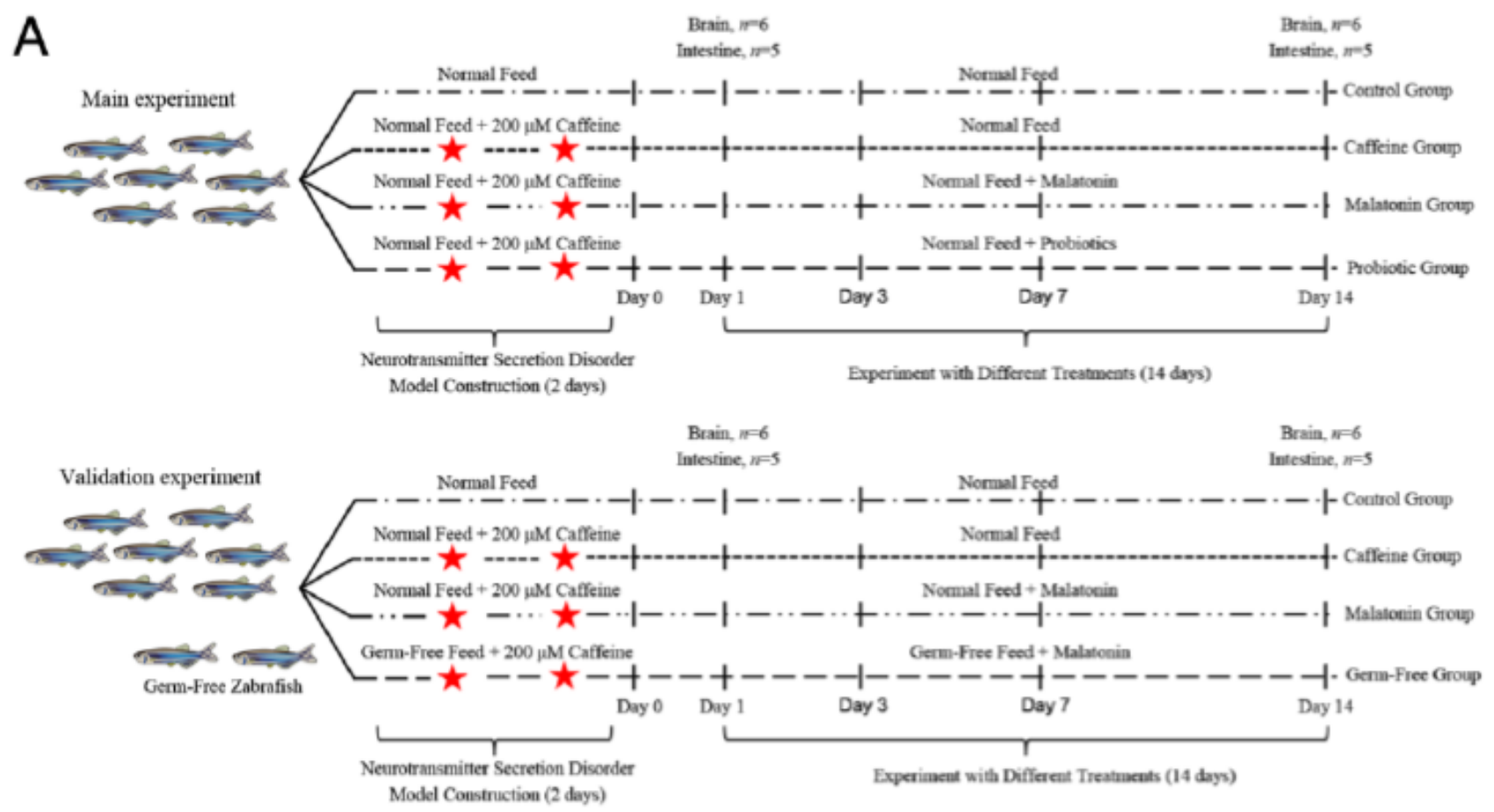

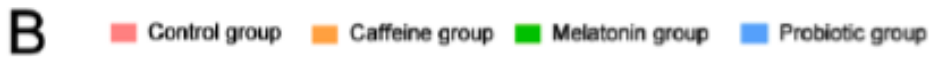
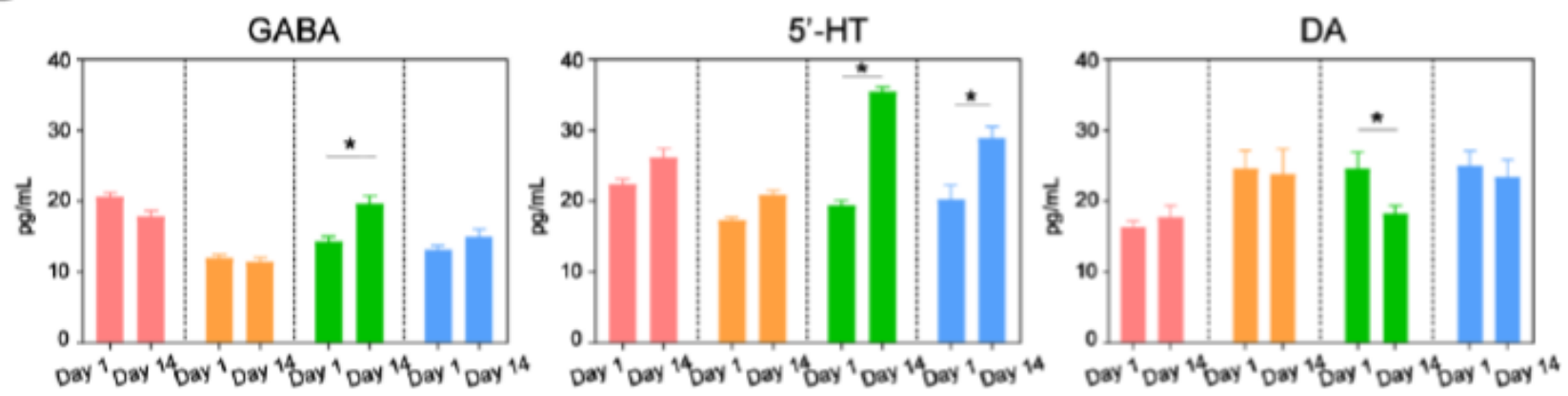

Figure 1 
(A) Experimental design. The main experiment, the fish was divided into four groups. The control group was treated with normal feed and regular filtered water throughout the experiment. The caffeine, melatonin and probiotic groups were subjected to two consecutive days of caffeine exposure and then maintained under the corresponding conditions (caffeine group: normal feed + regular filtered water, melatonin group: normal feed + filtered water with $1 \mu \mathrm{M}$ melatonin, probiotic group: feed containing 108 CFU Lactobacillus plantarum HNU082/g of feed + regular filtered water). On day 1 and day 14 after caffeine induction, the brain and intestine of each zebrafish were collected for neurotransmitter level determination and high-throughput sequencing, respectively. For the verification experiment, the study added the GF zebrafish groups, and the experimental design and feed conditions were the same as the main experiment. (B) Effects of neural hyperactivity on the levels of neurotransmitters (DA, $\mathrm{Y}$-GABA, 5-HT) in the zebrafish brain; the variation in the secretion levels of neurotransmitters in response to melatonin and probiotic supplementation were determined by ELISA. Mann-Whitney test and $t$ test were used to assess the differences in neurotransmitter levels in the zebrafish brain on day 1 and day 14 , respectively. $*, p<0.05$. 


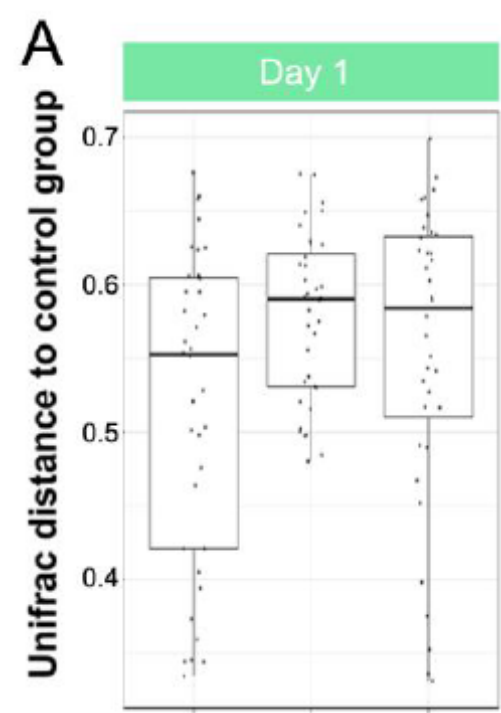

B Caffeine Melatonin Probiotic

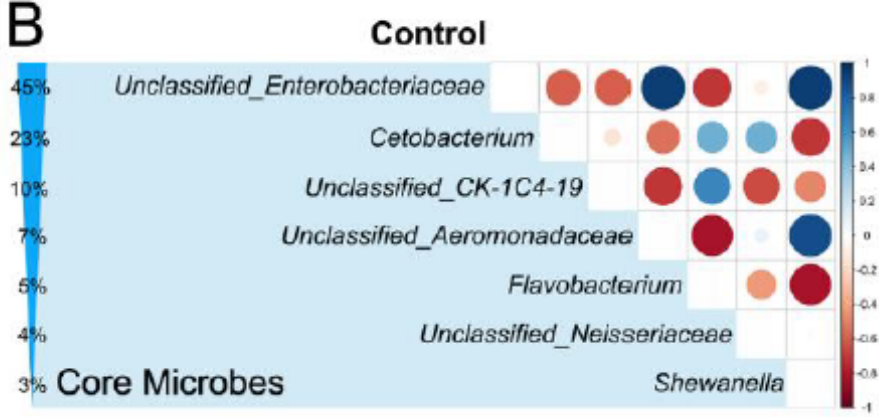

Melatonin

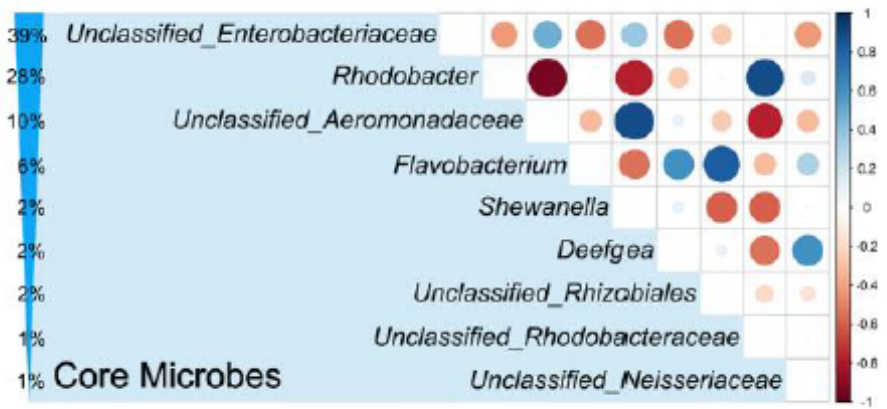

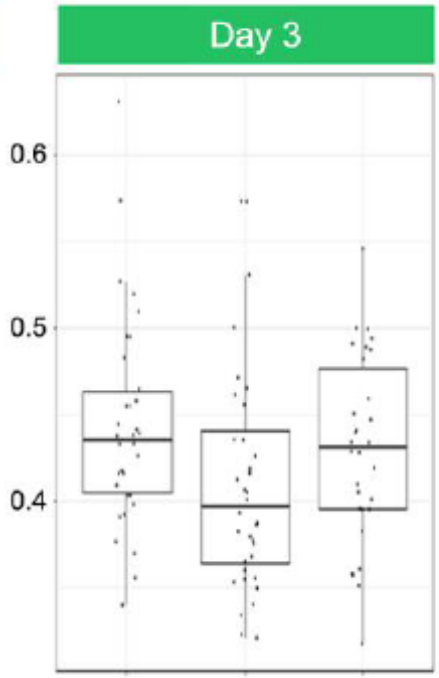

Caffeine Melatonin Probiotic
0.4

Caffeine Melatonin Probiotic

Day 14

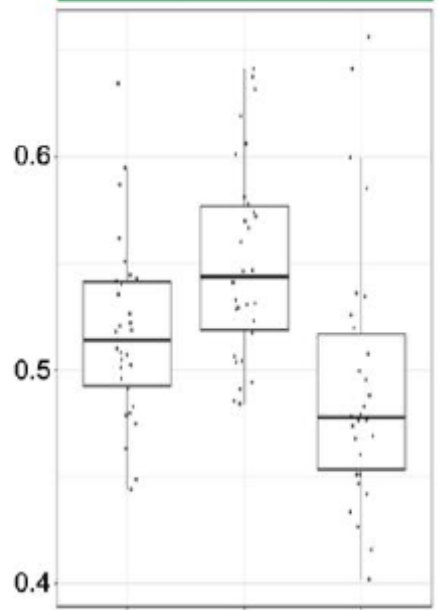

0.5

6

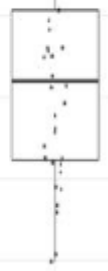

0.6

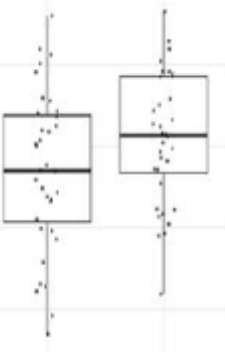

Caffeine Melatonin Probiotic

\section{Caffeine}

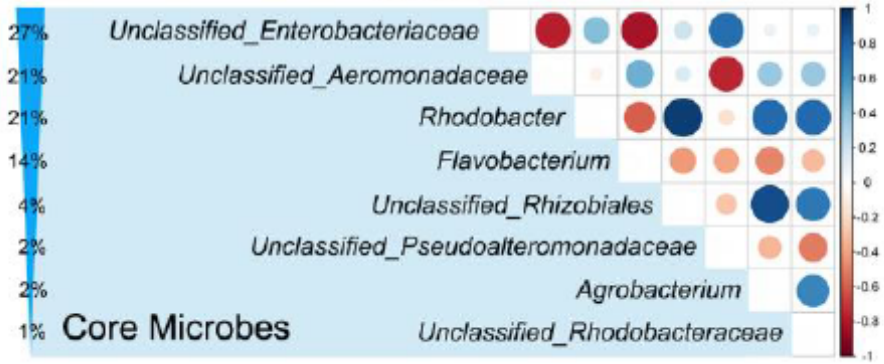

Probiotic

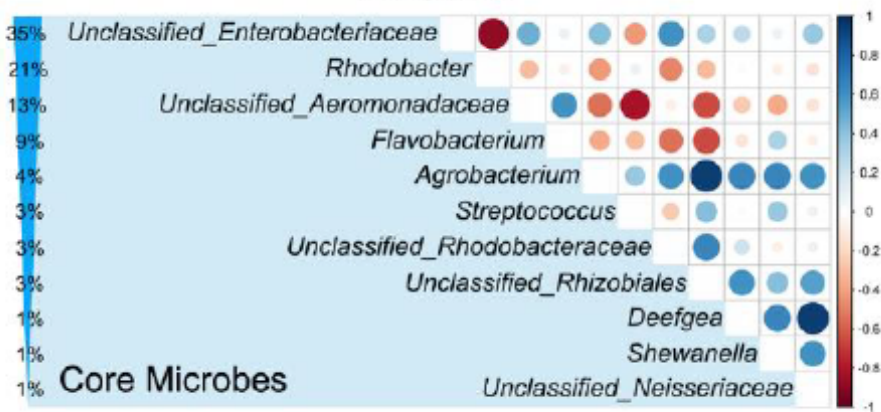

\section{Figure 2}

(A) The distance based on the unweighted UniFrac between the control and three different treatment groups display the difference of the intestinal microbiota among different groups on day 1 (A), day 3 (B), day 7 (C) and day 14(D), which include control vs caffeine, control vs melatonin, and control vs probiotic.

(B) Comparative analysis of core genera accounting for more than $1 \%$ in the control, caffeine, melatonin and probiotic groups after 14 days of corresponding treatment. The percentage indicates the relative abundance of the corresponding genus. The size and colour of the circle are proportional to the correlation intensity. The redder the color, the stronger the negative correlation, and the bluer the color, the stronger the positive correlation. 
A
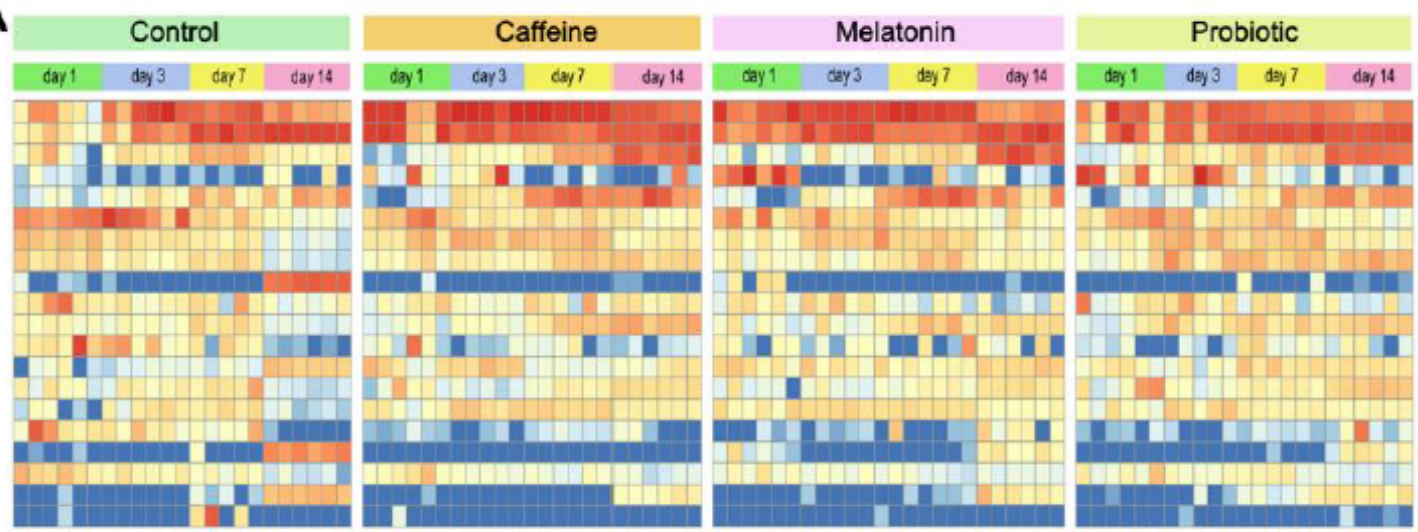

Difference genera

B
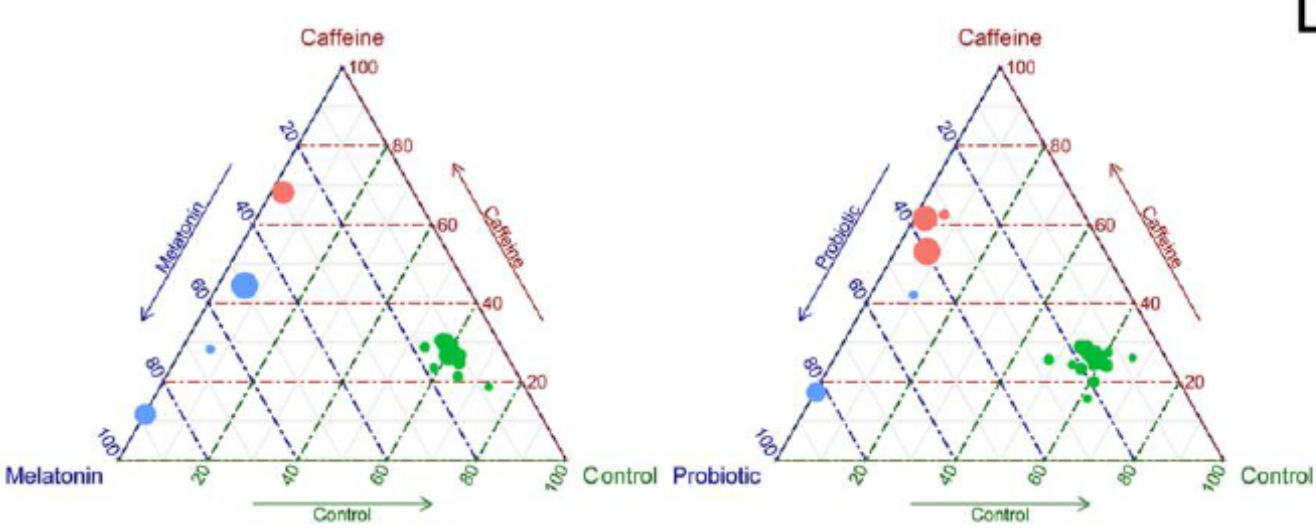

D

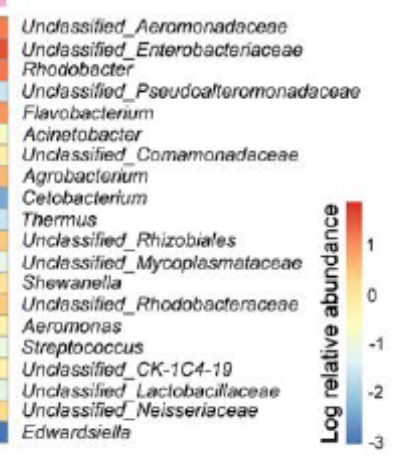

Day 1 CMI Day 1

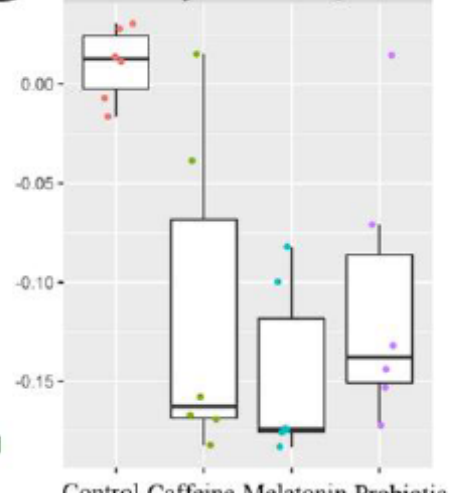

C
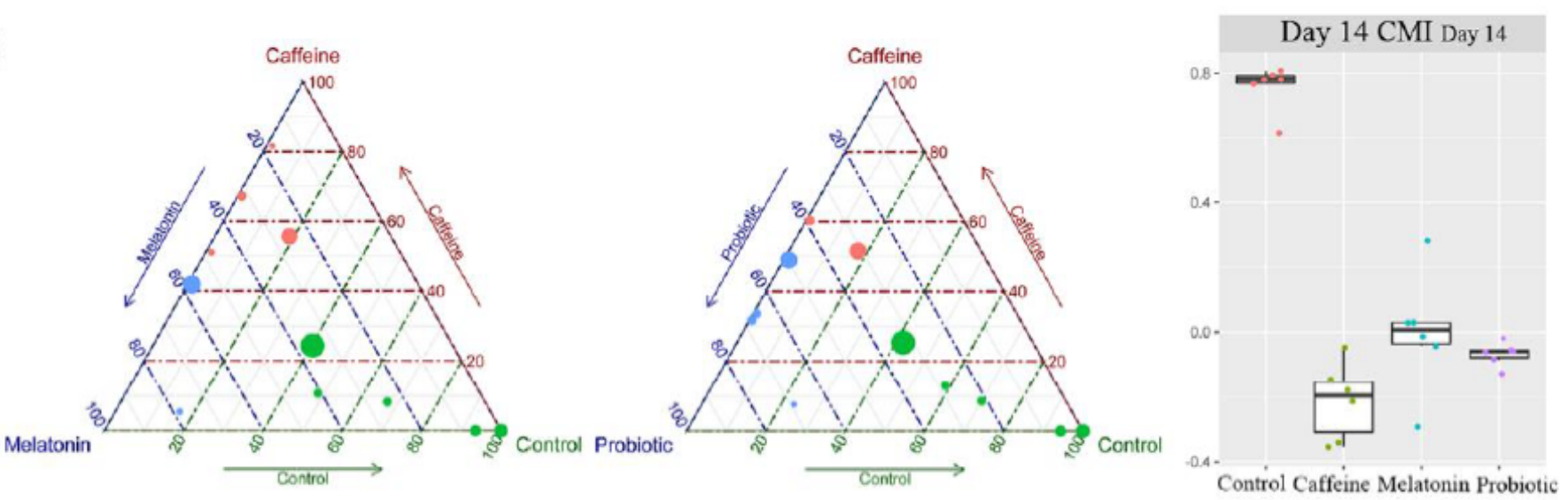

\section{Figure 3}

(A) The distance based on the unweighted UniFrac between the control and three different treatment groups display the difference of the intestinal microbiota among different groups on day 1 (A), day 3 (B), day 7 (C) and day 14(D), which include control vs caffeine, control vs melatonin, and control vs probiotic. (B) Comparative analysis of core genera accounting for more than $1 \%$ in the control, caffeine, melatonin and probiotic groups after 14 days of corresponding treatment. The percentage indicates the relative abundance of the corresponding genus. The size and colour of the circle are proportional to the correlation intensity. The redder the color, the stronger the negative correlation, and the bluer the color, the stronger the positive correlation. 
A

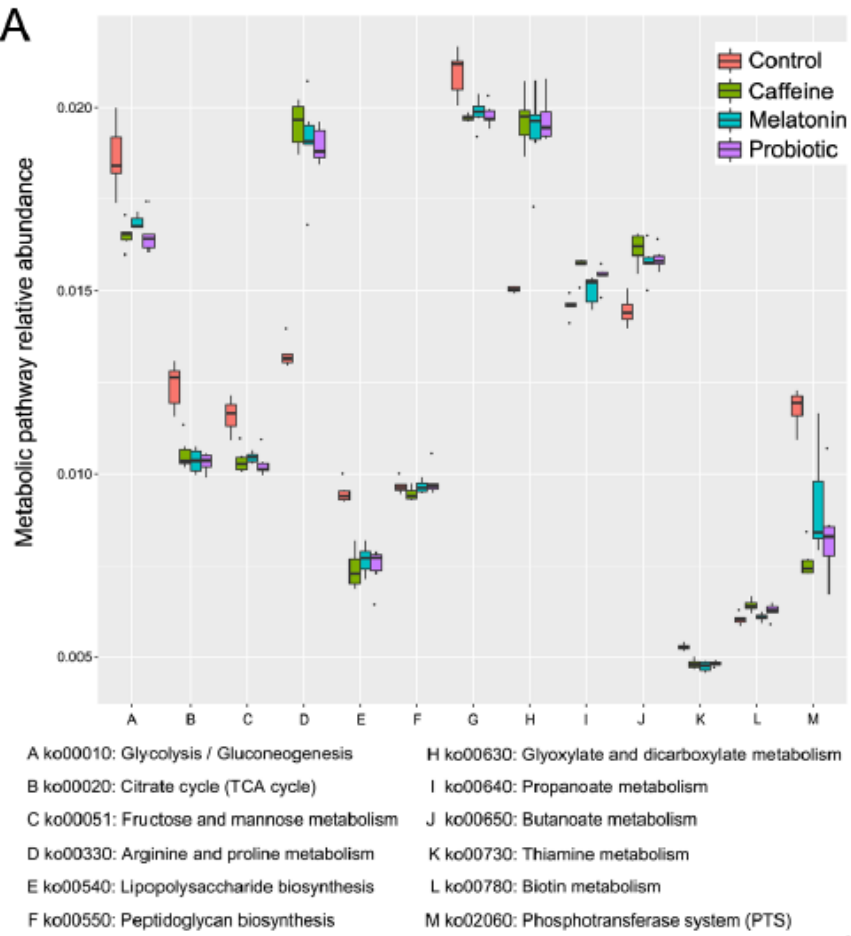

G ko00620: Pyruvate metabolism
B Microbial metabolic pathway analysis between Caffeine and Melatonin group on Day 14

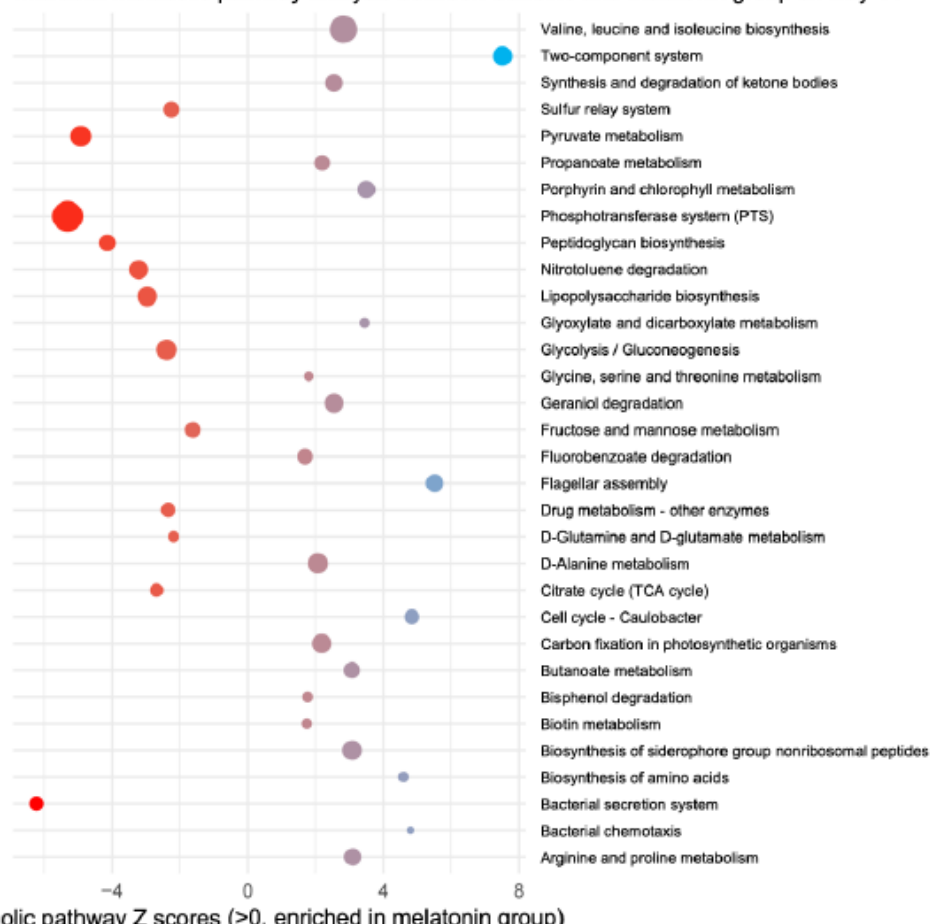

Figure 4

Differential functional features of the zebrafish intestinal microbiota in the caffeine, melatonin, probiotic and control groups after 14 days of corresponding treatment. Metabolic pathway analysis was performed based on the shotgun metagenomic sequencing data, which were compared with the KEGG database to further explain the microbial metabolic pathways. Z scores greater than 1.6 were selected as significant differences. 


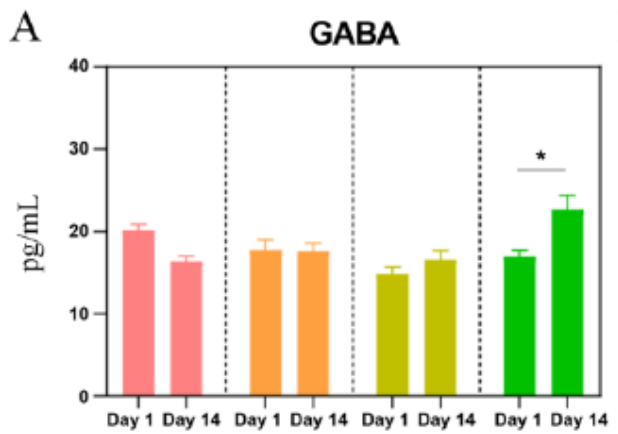

B

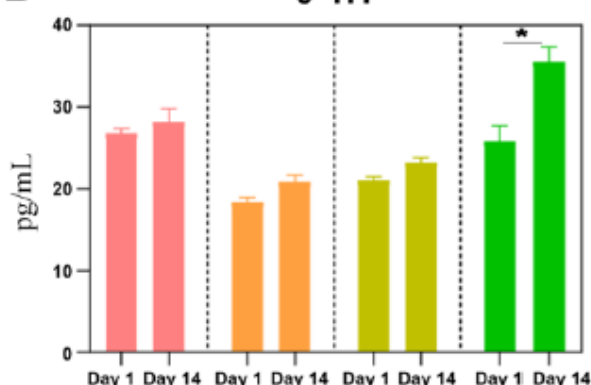

$\mathrm{C}$

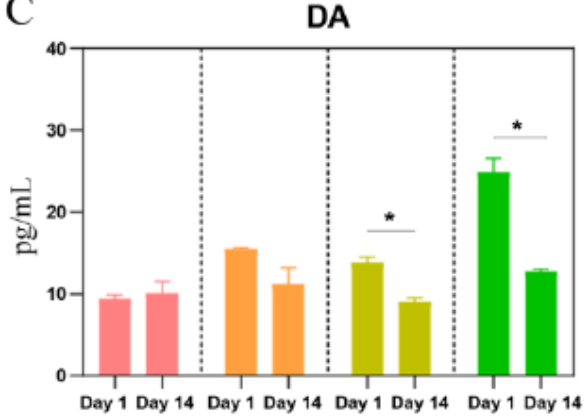

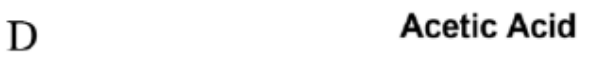
E Propionic Acid
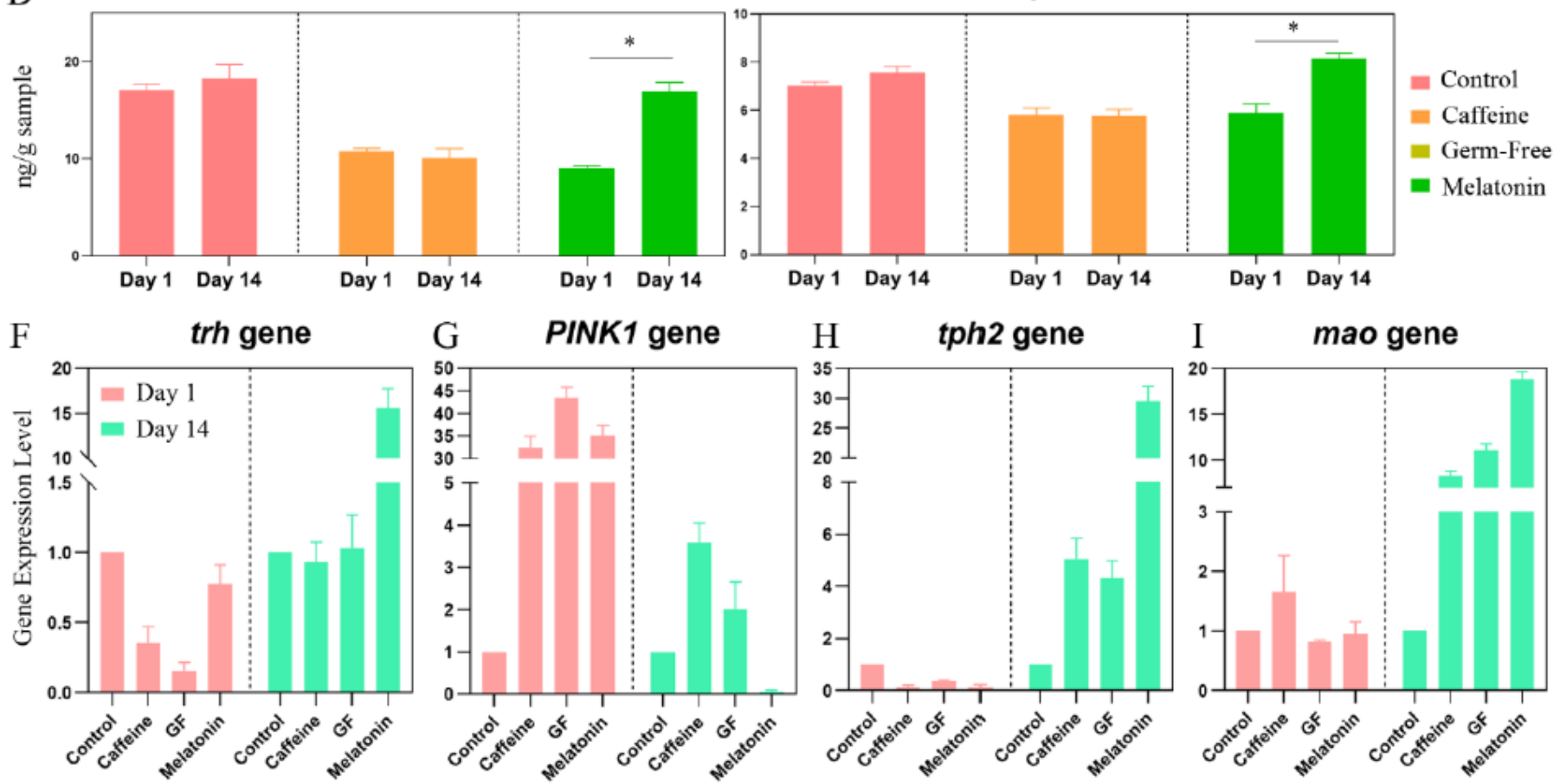

Figure 5

Further verification was performed using germ-free zebrafish, and the levels of neurotransmitters in the zebrafish brain, including DA, $\mathrm{Y}-\mathrm{GABA}$ and 5-HT, were detected on day 1 and day 14 among the control, caffeine, GF and melatonin groups (A-C). Meanwhile, the zebrafish genes associated with DA (PINK1), $Y^{-}$ GABA (trh) and 5-HT (tph2 and mao) secretion were selected and subjected to real-time PCR analysis (FI). Changes in zebrafish intestinal concentrations of acetic acid (D) and propionic acid E) in the control, caffeine, GF and melatonin groups on day 1 and day 14 were also analysed by GC-MS, and contents are expressed as nanograms per gram of intestine. 

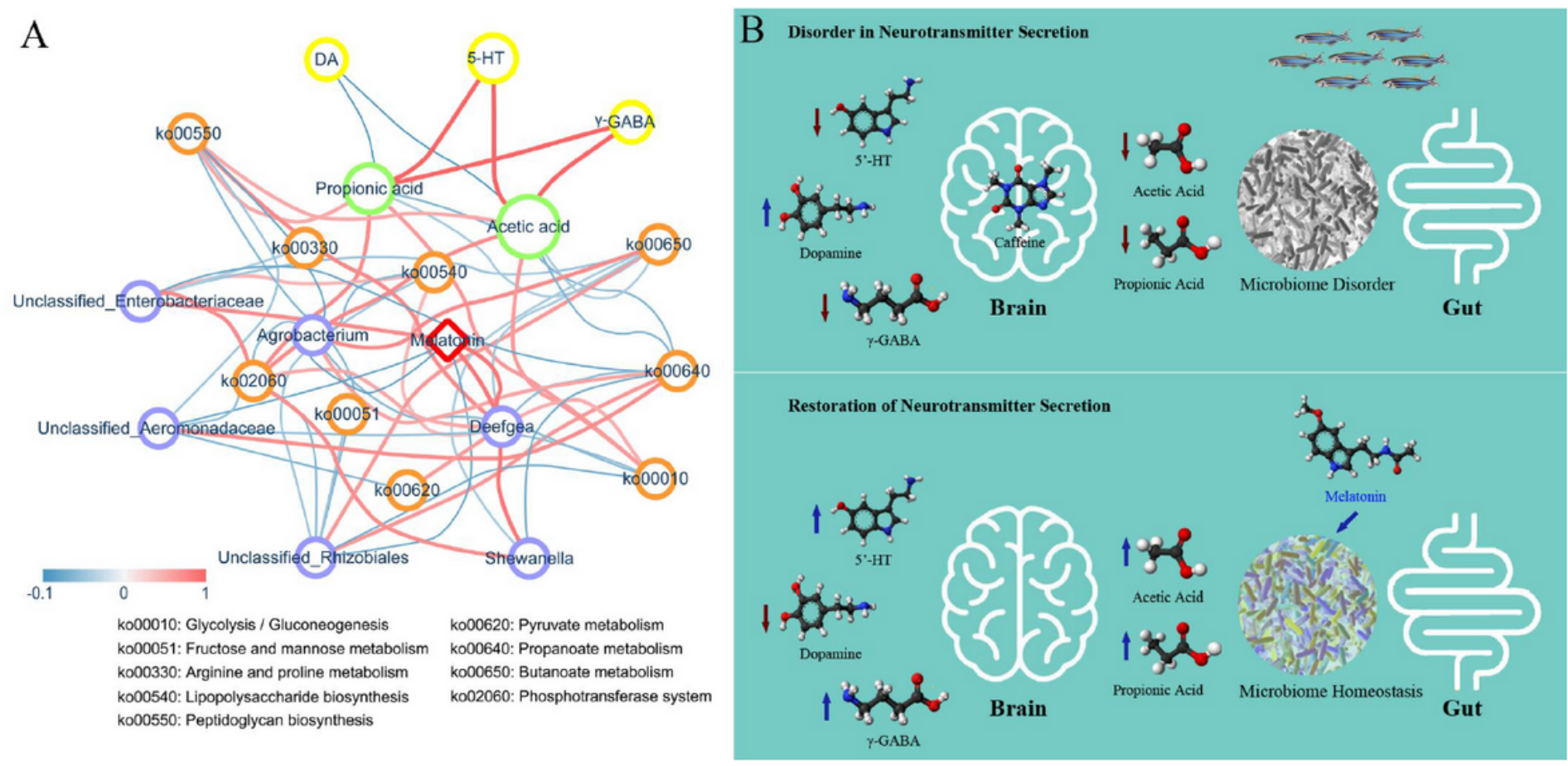

\section{Figure 6}

(A) The correlation network constructed among melatonin, differentially abundant genera, metabolic pathways, SCFAs and neurotransmitters (including DA, $\mathrm{Y}$-GABA and 5-HT) based on Spearman's rank correlation coefficient. An R value less than -0.4 or greater than 0.4 was selected. The width and colour (red indicates a positive correlation, while blue indicates a negative correlation) of the edge are proportional to the correlation intensity. The red node represents melatonin, blue nodes represent genera, orange nodes represent metabolic pathways, green nodes represent SCFAs, and yellow nodes represent neurotransmitters. The node size is proportional to the mean abundance in the respective population. (B) Summary diagram of the mechanism of melatonin regulation of neurotransmitter secretion disorders through the gut-brain axis.

\section{Supplementary Files}

This is a list of supplementary files associated with this preprint. Click to download.

- SupplementaryFIGTAB.pdf

- formulas.docx 